\title{
Percepción del alumnado universitario acerca del uso e integración de las TIC en el proceso educativo de la Facultad de Educación de Granada
}

\section{College students perceptions regarding the use and introduction if ICT in the educational process of the Faculty of Educational Sciences of Granada}

\author{
Marina Morales Capilla ${ }^{1}$, Ana María Ortiz Colón ${ }^{2}$, Juan Manuel Trujillo Torres ${ }^{3}$, Francisco \\ Raso Sánchez ${ }^{4}$ \\ ${ }^{1}$ Departamento Didáctica y Organización Escolar, Universidad de Granada, España (mmorales@ gmail.com) \\ 22Departamento de Pedagogía, Universidad de Jaén, España (aortiz@ujaen.es) \\ ${ }^{3}$ Departamento Didáctica y Organización Escolar, Universidad de Granada, España (jttorres@ugr.es) \\ ${ }^{4}$ Departamento Didáctica y Organización Escolar, Universidad de Granada, España (fraso@ugr.es)
}

Recibido el 8 de noviembre de 2015; revisado el 17 de noviembre de 2015; aceptado el 17 de noviembre de 2015; publicado el 14 de diciembre de 2015

\section{RESUMEN:}

La manera de poder adaptarse a la sociedad actual del conocimiento y la información es adaptarse a las exigencias y adoptar los beneficios que las TIC presentan, estableciendo la posibilidad de innovar y transformar los espacios académicos, así como las estrategias y materiales pedagógicos para hacer universitarios competentes frente a la sociedad actual y al mercado laboral.

En base a esto, el presente estudio, pretende conocer la percepción de alumnos de la Facultad de Ciencias de la Educación de la Universidad de Granada acerca del uso de las TIC en los procesos educativos en relación a la integración de dichas herramientas por parte del profesorado. Se empleó como instrumentos de recogida de datos el cuestionario y la entrevista, concluyéndose que la actitud ante la integración y uso de las TIC es bastante positiva, demandándose una mayor formación por parte del profesorado en herramientas virtuales.

PALABRAS CLAVE: ENSEÑANZA SUPERIOR, COMPETENCIAS DEL DOCENTE, PROCESO DE APRENDIZAJE, MÉTODO DE ENSEÑANZA, TECNOLOGÍA EDUCACIONAL.

\section{ABSTRACT:}

The best way to settle in the current society of knowledge and information, is to conform to the demands and assume the profits of ICT, fostering the possibilities to innovate and transform educational spaces, the same as teaching strategies and resources, in order to make competent graduates who can gain access to the labour market. According to this, the following research tries to discover the perception of students from the Faculty of Educational Sciences of Granada (Spain) about the use of ICT in educational processes depending on the way that teachers introduce these tools. We used enquiries and interviews to conclude that, next to the introduction use of ICT, their attitude was quite positive, demanding a better teacher training with respect to virtual tools.

$\begin{array}{lcr}\text { KEYWORDS: } & \text { HIGHER } & \text { EDUCATION, } \\ \text { TEACHER QUALIFICATIONS, } & \text { LEARNING } \\ \text { PROCESSES, } \quad \text { TEACHING } & \text { METHODS, } \\ \text { EDUCATIONAL TECHNOLOGY. } & \end{array}$




\section{I INTRODUCCIÓN}

La actual sociedad del conocimiento y la información en la que nos encontramos nos plantea un escenario en el que las Tecnologías de la Información y la Comunicación (TIC) desempeñan un papel de vital importancia en todos los ámbitos, incluida la educación. Las transformaciones que se están produciendo en la sociedad afectan al ámbito educativo, de forma que la manera de poder hacer frente a dichas transformaciones y exigencias es establecer la posibilidad de innovar y transformar los espacios académicos, las estrategias y materiales pedagógicos de manera que se posibilite dar respuesta a los desafíos que se plantean.

Estas nuevas estrategias serán un vehículo para poder alcanzar los objetivos planteados por el Espacio Europeo de Educación Superior (EEES) y, para que todo esto sea posible, es necesario que exista un cambio en la mentalidad del proceso educativo, así como en la metodología de enseñanza. Por consiguiente, tanto profesores como alumnos deberán adquirir roles diferentes, de manera que las competencias de ambos encajen con el nuevo perfil académico exigible por la sociedad. Estos cambios se basarán en un tipo de enseñanza centrado en el alumno, siendo este partícipe y principal protagonista del proceso educativo, llevándolo a cabo mediante metodologías activas y la utilización de recursos y técnicas que generen el conocimiento. La introducción de las TIC ofrecerá la posibilidad de adaptar el proceso educativo para formar un perfil de profesional que se adapte a las exigencias de la sociedad actual.

\subsection{Inserción de las TIC en la Educación Superior}

Las TIC, desde la Unión Europea, con la creación del EEES, se conforman como un recurso de gran importancia tanto para fomentar la actitud empresarial, ampliar la oferta formativa, llevar a cabo procesos administrativos, fomentar la investigación, innovación y aprendizaje significativo $\mathrm{y}$ formar a futuros profesionales competentes adaptados a las exigencias de la sociedad actual. El objetivo, como indican Ballesteros, Franco y Carañana (2012), es promover un conocimiento y una educación actualizada que pueda satisfacer las exigencias que en cada momento acontecen al mercado laboral.

Marquès (2001) señala cinco cambios importantes que nos ofrece la disponibilidad de las TIC: mayor universalización de la información, metodologías y enfoques crítico-adaptativos para el autoaprendizaje, actualización de los programas, trabajo colaborativo y construcción personalizada de aprendizaje significativos. De igual modo, Badía (2006) hace un recorrido por las ayudas educativas que las TIC aportan: apoyo a la comprensión de actividades de aprendizaje, planificación del aprendizaje, provisión de contenidos, apoyo a la construcción de conocimiento, comunicación y colaboración y evaluación del progreso de aprendizajes. Las TIC, por tanto, facilitan nuestros trabajos (Marquès, 2001), pero siempre requieren una cierta selección crítica y curación de contenido para realizarlos y comunicación con otras personas: acceso a todo tipo de información, proceso de datos de manera rápida y fiable $\mathrm{y}$ canales de comunicación inmediata, sincrónica y asincrónica para difundir la información y contactar con cualquier persona o institución. Entre estas, podemos encontrar otras numerosas posibilidades que las TIC ofrecen como la ruptura de las barreras espacio-temporales para la realización de actividades, tutorización virtual y contacto permanente para resolución de problemas, motivación, promover la reflexión, gestionar el conocimiento de la asignatura, flexibilizar los contenidos, individualizar el proceso de enseñanza, optimizar el uso del tiempo, posibilitan los procesos formativos abiertos y flexibles, rápido acceso a la información (Ferro, Martínez y Otero, 2009; Marquès, 2001; Santos, Galán, Izquierdo y Olmo, 2009).

A pesar de las posibilidades que las TIC ofrecen a la educación, no todo el profesorado se encuentra de acuerdo con la integración de estas en el proceso educativo. Existen barreras tecnológicas que impiden a los profesores apreciar la importancia de la incorporación de las TIC en el desarrollo de las clases (Araujo y Bermúdez, 2009). Estos autores concluyen en la necesidad de crear, dentro de las instituciones educativas, ambientes de aprendizaje que sean efectivos, de manera que se proporcione los recursos necesarios, siendo esto una responsabilidad de solicitud de toda la comunidad educativa. Cabero, Llorente, Leal y Andrés (2009), por su parte, señalan una serie de limitaciones que ofrecen las TIC como son el acceso a recursos necesarios, la necesidad de una infraestructura específica, apoyo técnico, necesidad de formación y adaptación y entornos poco estáticos y motivadores.

Las nuevas tecnologías y las exigencias del marco Europeo han supuesto la iniciación de numerosos cambios en cuanto a roles y competencias de los participantes en el proceso educativo, de manera que, tal y como asegura 
Rodríguez (2011), se debe contar con el apoyo y trabajo conjunto de las instituciones y ser conscientes de que estamos ante un nuevo paradigma en el que debemos participar todos.

\subsection{Alfabetización digital}

Salinas (1998) considera que la necesidad de integración de las TIC en el sistema educativo conlleva la unión de dos aspectos, la sensibilización y la formación del personal docente. Esto equivale a una formación continua de los profesores en ejercicio respecto a las nuevas tecnologías. Anteriormente el rol del profesorado se centraba en transmitir contenidos que el alumno debía incorporar de forma mecánica y se cuidaba la funcionalidad de los aprendizajes así como su proceso de adquisición por parte de los alumnos, pero ahora se deben adquirir además de los conceptos, contenidos de tipo procedimental (De Juanas y Fernández, 2008).

Valcárcel (2003) asegura que es obvio que el éxito hacia una convergencia europea no es posible si no existe un profesorado universitario capaz y motivado que se ajuste a un perfil profesional que le permita responder adecuadamente a los nuevos retos y demandas que se plantean. Con la convergencia en el marco europeo de educación superior, se le pide al profesorado universitario un perfil basado en competencias y una nueva práctica docente basada en el conocimiento de las metodologías y didácticas activas, habilidades y destrezas (Trujillo, 2006).

El modelo de aprendiz que requiere este estado de convergencia europea se basa, según De la Cruz (2003), en los siguientes requisitos: el alumno será un aprendiz activo, autónomo, estratégico, reflexivo, cooperativo, un aprendiz responsable. En todo momento se producirá un proceso de comunicación entre el profesor y el alumno donde el primero proporciona retroalimentación (feedback) a los alumnos (Cruz, 2005).

Uno de los principales problemas de la alfabetización digital y de la incorporación de las TIC en el sistema educativo para que forme parte del proceso de enseñanza-aprendizaje y sobre todo para la incorporación de las TIC en la enseñanza universitaria, es la formación del docente en nuevas tecnologías (Cabero, 2010). La tecnología debe integrarse en todo programa de formación docente, de manera que los profesores universitarios sean competentes en TIC y puedan volcar sus saberes en los alumnos y futuros docentes, de esta forma estos últimos podrán enseñar a sus alumnos ciertas competencias en nuevas tecnologías que les serán útiles en la sociedad en la que nos encontramos. Por otro lado, deberán ser instruidos en crear actividades mediante recursos tecnológicos, que puedan llevar a la práctica en su futuro ámbito profesional.

\section{METODOLOGÍA}

El diseño utilizado se encuentra enmarcado en una investigación no experimental, basada en una metodología descriptiva e inferencial por encuesta (Buendía, 2001). Se lleva a cabo, en este sentido, una encuesta de diseño trasversal (Shaughnessy, Zechmeister, y Zechmeister, 2007), que ha sido sometida a un juicio de expertos, en el que se valora la fiabilidad (Fox, 1981) y la validez de contenido de dicha herramienta.

La metodología seguida fue basándose en el metanivel cuantitativo, por considerar que nos proporcionará la información relevante que precisamos para llegar a las conclusiones. Se aproxima a un planteamiento que recoge un interés descriptivo-ecléctico, utilizando para ello instrumentos de corte cuantitativo, como es el cuestionario, de manera que se pudo obtener la información relevante y precisa para llegar a las conclusiones de la investigación, contando así con la mayor y mejor cantidad de información posible.

\subsection{Objetivos}

En este trabajo se pretende conocer la utilización de las Nuevas tecnologías (TIC) en el sistema de educación superior:

- Percepción del alumnado de la Facultad de Ciencias de la Educación de la Universidad de Granada acerca del uso que se hace de las TIC en sus procesos de enseñanza-aprendizaje

- Percepción del alumnado de la Facultad de Ciencias de la Educación de la Universidad de Granada acerca de la integración y utilización de las TIC por parte del profesorado

- Analizar, por curso y especialidad del alumnado, la posible existencia de diferencias estadísticamente significativas entre las valoraciones.

De esta manera, se pretende detectar sus opiniones mejorando así la enseñanza y el proceso de aprendizaje en función a los aspectos que los alumnos demandan, teniendo en cuenta aquellos aspectos que se deben mejorar, así como las posibilidades de reestructurar la enseñanza en función de las necesidades formativas de los agentes educativos. 


\subsection{Descripción de la población y muestra}

Se toma por tanto como contexto la Facultad de Ciencias de la Educación de la Universidad de Granada. El universo o población a la que fue dirigida la investigación son todos los alumnos matriculados en los diferentes grados de la Facultad en el curso 2012-2013, ascendiendo estos a 3193 estudiantes. La muestra representativa que se obtiene para un análisis de dichos datos es a través de un muestreo aleatorio estratificado mediante el algoritmo de Buendía (2001) que arroja como resultado 343 sujetos, quedando la muestra representativa estratificada por niveles de la siguiente forma:

Tabla 1: elaboración propia. Distribución de los alumnos/as en grados y cursos

\begin{tabular}{cccccccc}
\hline GRADO & Alumnos & $1^{\circ}$ Grado & $1^{\circ}$ participante & $2^{\circ}$ Grado & $2^{\circ}$ participante & $3^{\circ}$ Grado & $3^{\circ}$ participante \\
\hline Ed. Primaria & 1778 & 528 & 57 & 580 & 62 & 670 & 72 \\
Ed. Infantil & 723 & 230 & 25 & 232 & 25 & 261 & 28 \\
Ed. Social & 368 & 111 & 12 & 111 & 12 & 146 & 16 \\
Pedagogía & 324 & 116 & 12 & 97 & 10 & 111 & 12 \\
\hline
\end{tabular}

\subsection{Instrumentos de recogida de datos}

El diseño utilizado se encuentra enmarcado en una investigación no experimental, basándonos en una metodología por encuesta. Consiste en un cuestionario (PIT-01) utilizando ítems de elección múltiple en una escala de respuesta de tipo Likert con cuatro opciones de respuesta e integrado por 87 variables, estructurado en 5 subescalas.

- Parámetros de identificación personal/profesional. (PIT-01 A).

- Valoración sobre la importancia de las TIC en educación (PIT-01 B1).

- Valoración sobre las posibilidades que ofrecen las TIC en el proceso de enseñanza-aprendizaje (PIT-01 B2).

- Valoración de los recursos TIC que utiliza el profesorado (PIT-01 C1).

- Valoración del alumnado sobre organización, metodología y contenido de las asignaturas (PIT$01 \mathrm{C} 2$ ).

La estructura de dicho cuestionario responde a una escala Likert con una graduación de respuesta de 1 a 4. Siendo: 1 (Totalmente en desacuerdo), 2 (En desacuerdo), 3 (De acuerdo), 4 (Totalmente de acuerdo).

El cuestionario (PIT-01) fue sometido a un grupo de expertos 10 expertos: cinco profesores del Departamento de Métodos de Investigación y Diagnóstico en Educación de la Universidad de Granada, y cinco profesores del Departamento de Didáctica y Organización Escolar de dicha Universidad, especialistas todos ellos en materia de Tecnología Educativa y Orientación Escolar. Del resultado de dicha valoración se aplicaron modificaciones al instrumento que darían lugar a su configuración definitiva para su aplicación. Al objeto, por otra parte, de medir el grado de consistencia interna y fiabilidad del PIT-01, se utilizó como parámetro de coeficiente $\alpha$ de Cronbach, cuyo valor, tanto total como por subescalas, podemos apreciar en la tabla 2.

Para el cuestionario de los alumnos, el índice de fiabilidad es el siguiente:

\begin{tabular}{|c|c|}
\hline Alfa de Cronbach & N de elementos \\
\hline, 941 & 87 \\
\hline
\end{tabular}

El resultado $\alpha=0,941$ en la prueba Alfa de Cronbach, es considerada muy buena, indicando una muy alta fiabilidad del instrumento (precisión alta). De esta manera, desglosamos la prueba Alfa de Cronbach, según los diferentes bloques de ítems para comprobar la fiabilidad.

Tabla 2: elaboración propia. Índice de fiabilidad de las variables objeto de estudio

\begin{tabular}{ccccc}
\hline & \multicolumn{2}{c}{ BLOQUE B } & \multicolumn{2}{c}{ BLOQUE C } \\
\hline & B1 & B2 & C1 & C2 \\
Alfa de &, 860 &, 877 &, 876 &, 893 \\
$\begin{array}{c}\text { Cronchach } \\
\text { Número de } \\
\text { variables }\end{array}$ & 22 & 20 & 21 & 21 \\
\hline
\end{tabular}

De esta manera queda constatada la fiabilidad alta del instrumento cuantitativo destinado a los alumnos para esta investigación.

\section{RESULTADOS}

\subsection{Datos cuantitativos}

En cuanto al análisis realizado sobre los datos del cuestionario de alumnos, se destacan las cuatro 
dimensiones en las que se dividen, de tal manera que:

Primer bloque: Percepción del alumnado acerca del uso que se hace de las TIC en sus procesos de enseñanza-aprendizaje.

Dimensión: valoración del alumnado acerca de la importancia de las TIC en educación.

Entre el $51,6 \%$ y el $34,7 \%$ del alumnado encuestado posee una actitud favorable ante la integración de las TIC en el proceso educativo encontrándose de acuerdo o totalmente de acuerdo al respecto. El cambio de metodología queda patente en las universidades, ya que se llevan a cabo formas de enseñanzas significativas, activas, en las cuales el alumno se involucra más en el proceso tanto de la enseñanza como del aprendizaje. Por tanto, el alumnado considera necesaria en un $43,8 \%$ y un $47,6 \%$ el cambio de metodologías tradicionales hacia procesos de enseñanza más activos, presentando la misma opinión en cuanto a la necesidad de adoptar un papel activo en los procesos de enseñanza-aprendizaje, con un $35,8 \%$ y $57,8 \%$ de opinión aceptante. Según la opinión del $86 \%$ de los encuestados, las TIC proporcionarán que el alumno desempeñe ese rol activo necesario para llevar a cabo procesos de enseñanza adecuados. En cuanto a las mejoras que ofrecen las TIC con respecto a la educación, los porcentajes de la mejora de la práctica profesional, la mejora de la calidad del aprendizaje y la mejora de los resultados académicos, se muestran los siguientes porcentajes de acuerdo-desacuerdo del alumnado.

Entre el $53,8 \%$ y el $35,3 \%$, considera que la utilización de las TIC posibilita la mejora de la práctica profesional. De igual modo, el 60,1\% de los alumnos considera que a través de la utilización de las TIC se mejora la calidad del aprendizaje, y un $21,7 \%$ presentan una opinión en total acuerdo con respecto a esta afirmación.

Acerca de la opinión de que el uso de las TIC mejora los resultados académicos se presentan discrepancias, no pudiendo posicionar la opinión de los alumnos en ninguna opinión concreta, ya que, aunque el mayor porcentaje, un 46,6\% están de acuerdo con que las TIC podrían mejorar los resultados académicos, el 38,7\% de los encuestados considera que no es así.

\section{El uso de las TIC mejoran los resultados academicos}

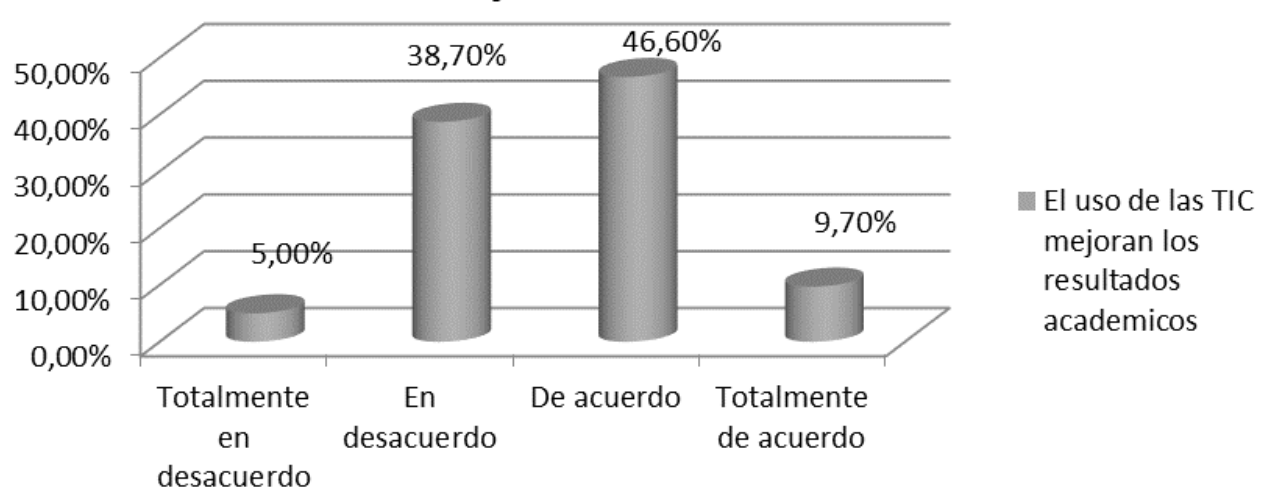

Gráfico 1: Valoración acerca de si el uso de las TIC mejora los resultados académicos

Con respecto a las facilidades que las TIC posibilitan al alumnado en sus procesos de aprendizaje, se pueden destacar los siguientes resultados: en su mayoría, con un $59,2 \%$ y un $26,5 \%$, los alumnos consideran que la utilización de las TIC facilita la construcción del conocimiento, debido a la gran cantidad de herramientas que ofrecen. Un $1,2 \%$ de los alumnos encuestados consideran que las TIC no facilitan en ningún caso la construcción del conocimiento a aprender. De igual modo, un porcentaje de un $59,2 \%$ considera que el aprendizaje que se realiza mediante la utilización de las TIC es un aprendizaje significativo, en el que los alumnos abandonan el método repetitivo de memorización, y son ellos los que construyen su propio conocimiento, comprendiendo y completando el proceso, conociendo lo que significa cada paso del proceso de aprendizaje. 


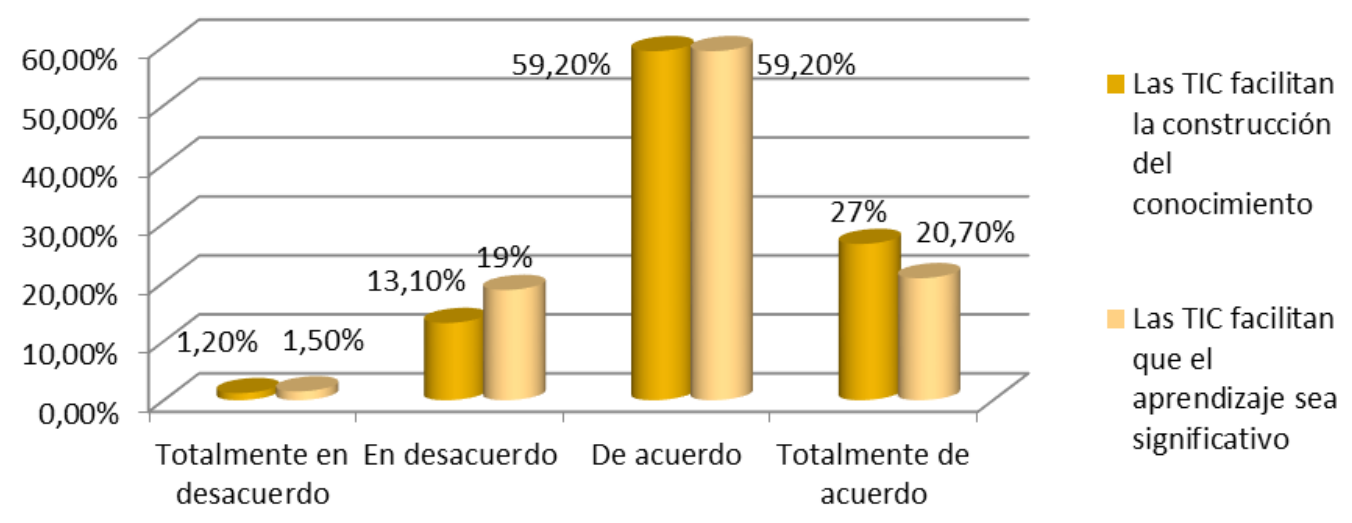

Gráfico 2: Valoración sobre las facilidades que ofrecen las TIC ante el aprendizaje

A pesar de ser una metodología activa, con un cambio en el rol del alumnado y ellos estar muy de acuerdo con la necesidad de este cambio y a favor de las numerosas posibilidades que ofertan, un $54,6 \%$ de los alumnos encuestados consideran que las TIC ofrecen mayor responsabilidad al alumnado en el proceso de enseñanza-aprendizaje. El uso de las TIC proporciona una enseñanza más especializada y personalizada dedicada a cada alumno y a sus necesidades de aprendizaje. Con respecto a ese tema, el $54,4 \%$ de la población encuestada está de acuerdo con esta afirmación, mientras un 3,2\% está totalmente en desacuerdo. Es por otro lado, que los alumnos reclaman que mayor número de profesores deben utilizar las TIC en el proceso de enseñanza-aprendizaje, ya que ofrecen grandes posibilidades para el aprendizaje y son recursos de gran importancia. Así el 49,9\% y el
$39,7 \%$ de los alumnos encuestados están de acuerdo y totalmente de acuerdo con la necesidad de que los profesores utilicen las TIC de forma generalizada. De tal modo, la formación en TIC por parte del alumnado y del profesorado es un tema que está expuesto a debate, y por consiguiente se quiere saber la opinión del alumnado respecto al tema. El alumnado es el principal afectado, de manera que los resultados de los ítems con respecto al tema son los siguientes: con respecto a la formación del profesorado en TIC, se presenta un porcentaje de un $32,9 \%$ y $63 \%$ de alumnado que está de acuerdo y totalmente de acuerdo con esta formación del profesorado. Es por tanto, por lo que igualmente consideran necesario que el alumnado debe formarse inicialmente en Nuevas Tecnologías, presentando unos porcentajes del $46,4 \%$ y $44,7 \%$.

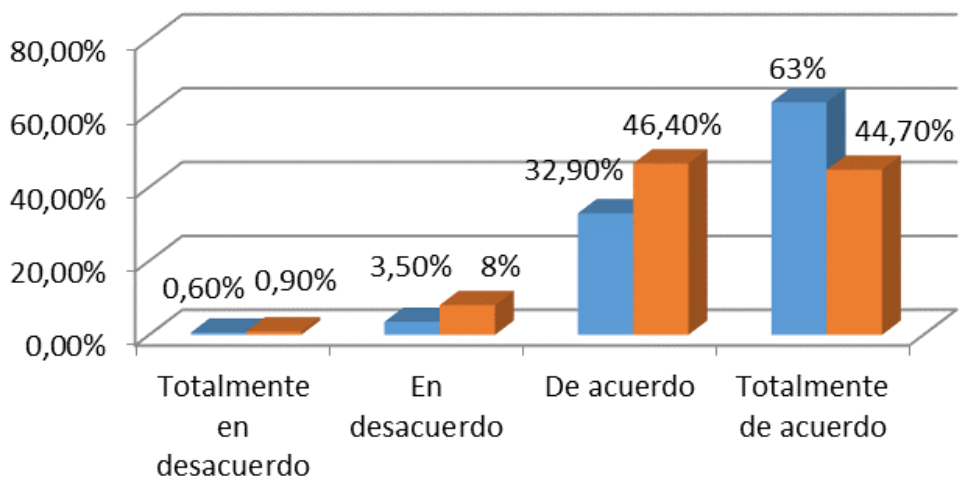

\author{
- Necesaria la \\ formación en TIC \\ por parte del \\ profesorado \\ - Necesaria la \\ formación inicial \\ en TIC por parte \\ del alumnado
}

Gráfico 3: Valoración sobre la necesidad de formación en TIC por parte del profesorado y del alumnado

En cuanto al uso que el profesorado hace de las TIC en los procesos de enseñanza-aprendizaje, la mayoría de alumnos están de acuerdo con que el uso es deficiente como asegura un $40,8 \%$, encontrándose muy reñida la opinión de estar en desacuerdo y totalmente de acuerdo con un $27,7 \%$ y un $26,2 \%$.

A continuación se procede a mostrar los resultados, más destacados, del análisis de contingencias del alumnado de sus respectivos 
cruces entre variables. La prueba que se utilizó es la prueba Chi-Cuadrado (Pearson) y su significatividad asintótica bilateral de Lilliefors. El nivel de confianza con el que se trabaja en este caso es del $95 \%(\alpha=0,95, \mathrm{p}<0,05)$.

En este ámbito del cuestionario, el análisis de las variables de grado /especialidad y la valoración sobre la importancia de las TIC en educación, muestra que un 50,8\% del alumnado de Primaria es el que más de acuerdo se encuentra ante una necesidad de cambio de metodología tradicional hacia una actuación docente más activa para desarrollar el proceso educativo, frente a un 1,5\% del grado de pedagogía que se encuentra en desacuerdo. El 39,6\% del alumnado del grado de primaria, presenta un porcentaje mayoritario frente a que existe un uso deficiente de las TIC por parte del profesorado. Cabe destacar al alumnado del grado de Pedagogía que no ha manifestado su total desacuerdo en este caso particular, distribuyéndose en mayor medida entre las valoraciones de contento. Es destacable en función del corte por cursos y la relevancia del uso de las TIC en el proceso educativo que existen diferencias estadísticamente significativas, estimando que el $33,2 \%$ de los alumnos de tercer curso están de acuerdo en algún grado con dicho supuesto. El resto de cursos, en su mayoría, muestran su contento con dicho aspecto en proporciones similares. Se destaca el alumnado de tercero que no manifiesta en ningún grado su total descontento. Al igual que en el caso anterior, el $32,3 \%$ del alumnado de tercer grado, es aquel que valora positivamente la necesidad de la utilización de las TIC ya que mejora la calidad de aprendizaje. Con respecto a uso deficiente por parte del profesorado ante el uso de las TIC, el alumnado de tercer curso, en un 28,3\%, muestra un mayor porcentaje de acuerdo ante dicha situación, siendo los alumnos pertenecientes a primer curso que se muestran en un $15,5 \%$, de acuerdo e igualmente en otro $15,5 \%$ en desacuerdo.
Dimensión: valoración sobre las posibilidades que ofrecen las TIC en el proceso de aprendizaje

Con respecto a la búsqueda de información, el aprendizaje y compresión del contenido, los alumnos encuestados dan la siguiente valoración de las posibilidades que las TIC ofrecen al respecto: Se aprecia una valoración muy alta, del $77,7 \%$ de los alumnos encuestados que se encuentra totalmente de acuerdo el hecho de que las TIC facilitan y posibilitan la búsqueda de información, esto es debido a los numerosos buscadores y a la amplia oferta informativa que ofrece la red, cuya finalidad es proveer al estudiante de diferentes instrumentos que le sirvan de apoyo para el proceso de aprendizaje. De igual modo, se observa una valoración muy positiva del $56,2 \%$ y $29,7 \%$ en cuanto a que la utilización de las TIC facilita que se contraste la información y contenidos encontrados en la red y permiten de igual modo seleccionar aquella que se considera más relevante para el aprendizaje que acontece. Llama la atención, la valoración de un $0 \%$ de aquellos alumnos que se encuentra en total desacuerdo ante dicha información. Ante una opinión muy destacada del alumnado en función al 88,7\%, los alumnos sienten que mediante la utilización de las TIC, poseen una mayor autonomía ante su proceso de aprendizaje, ya que son ellos los responsables de la creación, búsqueda y selección de los contenidos. El alumno, al desempeñar un rol totalmente activo en la búsqueda y formación del contenido y de su aprendizaje, establecen que se convierten en responsables de su propio aprendizaje y se siente motivados e implicados en el proceso, dedican más tiempo a trabajar ya que están permanentemente activos al interactuar con el ordenador, contando con una opinión del 47,6\% y 39,1\% ante la opinión de presentar mayor motivación e implicación en el proceso de aprendizaje.

Cabe destacar la comunicación que se establece entre compañeros y para/con el profesor/tutor. Las TIC facilitan esa conexión, así... 


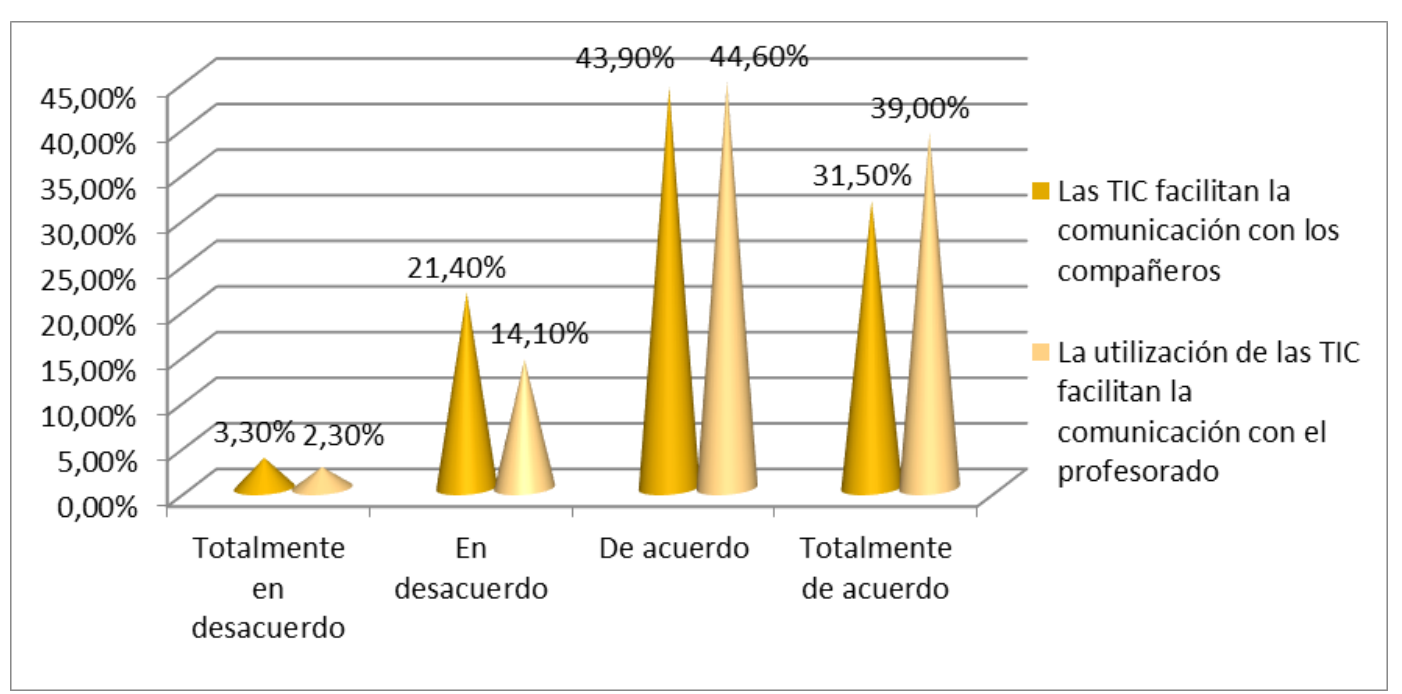

Gráfico 4: Valoración acerca de la facilidad que ofrecen las TIC ante la comunicación entre los compañeros y la comunicación con el profesorado

Debido a la facilidad de comunicación entre los miembros del proceso de enseñanza-aprendizaje, las TIC dan lugar a que se lleve a cabo un trabajo colaborativo, de manera que permite intercambiar experiencias y opiniones entre los compañeros y profesorado a través de canales de comunicación de manera sincrónica y asincrónica permitiendo difundir la información y contactar con cualquier persona. Entre el $46,5 \%$ y el $25,9 \%$ del alumnado encuestado valora de forma positiva que la utilización de las TIC fomenta el trabajo colaborativo, posibilitando el intercambio de experiencias y opiniones, siendo este hecho valorado, igualmente, de forma positiva, por el $53,1 \%$ y $34,9 \%$ del alumnado. Ahora es posible romper con los clásicos escenarios. A través de herramientas tecnológicas como simuladores el alumno podrá comprender la realidad con la ayuda de las TIC. De este modo, la comprensión de la realidad a través de las TIC lo valora positivamente un $67,3 \%$. Así...

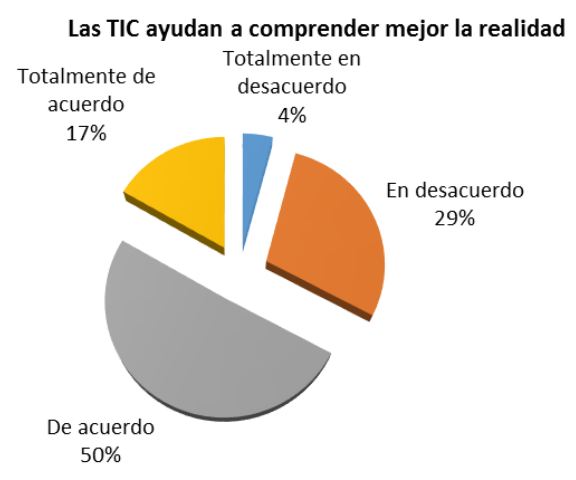

Gráfico 5: Valoración acerca de si las TIC ayudan a comprender mejor la realidad
Acerca de la valoración sobre las posibilidades que las TIC ofrecen en el proceso de aprendizaje, nos encontramos que no existen diferencias estadísticamente significativas en función de los diferentes grados o especialidades, al igual que ocurre con el corte por cursos, exceptuando un ítem. El alumnado de tercer curso, con un 30,6\%, muestra su contento en algún grado con que el uso de las TIC fomentan el trabajo colaborativo. Se destaca, por consiguiente, al alumnado de segundo curso que le sigue en proporción con un $22,3 \%$ en grado de acuerdo o total acuerdo.

Segundo bloque: Percepción del alumnado acerca de la integración y utilización de las TIC por parte del profesorado

Dimensión: valoración de los recursos TIC que utiliza el profesorado

El 74\% considera que el profesorado hace uso de las TIC en algún momento del proceso de enseñanza, pero la impartición de nociones elementales acerca de los recursos que se van a utilizar, es una opinión muy dividida entre el alumnado, ya que el $50,7 \%$ considera que no se ofrecen nociones elementales acerca de la utilización de los recursos que se van a usar, frente a un $49,3 \%$ de los encuestados que considera que el profesorado sí proporciona nociones elementales acerca de dichos recursos. Por otro lado, el 63,9\% está a favor de que los recursos tecnológicos que se utilizan no presentan ningún tipo de dificultad en su manejo, por lo que los alumnos suelen desenvolverse bien en cuanto al manejo de las TIC que toman parte en los procesos de enseñanzaaprendizaje. Para la distribución del contenido, el 
profesorado utiliza las TIC, así como para la exposición de los mismos en clase. En este sentido, se cuenta con un $80,1 \%$ y un $87,6 \%$ a favor de ambas afirmaciones respectivamente. De igual modo, el profesorado motiva a sus alumnos en la búsqueda de información e investigación a través de la red. La opinión del alumnado en cuanto a la enseñanza por parte del profesorado de recursos tecnológicos aplicables a una posterior práctica profesional, se encuentra muy dividida, estando un $53,4 \%$ en desacuerdo con la afirmación planteada, frente a un $46,6 \%$ de la muestra que está a favor de que el profesorado sí enseñe esta materia.

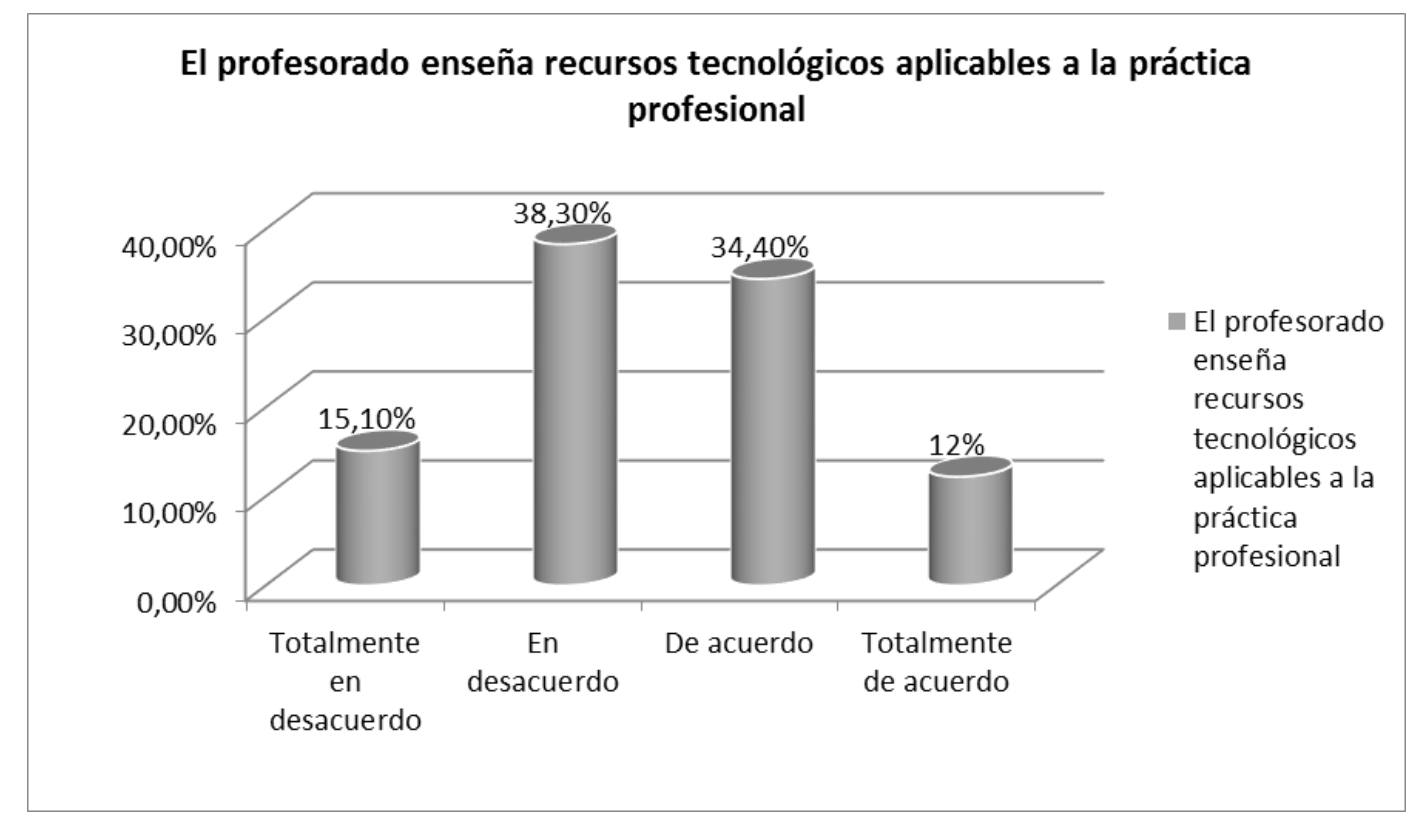

Gráfico 6: Valoración sobre si el profesorado enseña recursos tecnológicos aplicables a la posterior práctica profesional

La utilización de herramientas virtuales en el proceso de enseñanza-aprendizaje conlleva, también, que el alumno aprenda sobre los diferentes soportes existentes para poder elegir el más adecuado a su proceso educativo. Es por lo que el profesorado debe fomentar en los alumnos la búsqueda e investigación sobre TIC. En este sentido, el $46,9 \%$ considera que el profesorado no fomenta en los alumnos esta búsqueda e investigación, frente al $53,1 \%$ que considera que si se hace. La utilización de las TIC en el proceso de enseñanza-aprendizaje permite la adquisición de competencias tecnológicas por parte del alumnado, útiles para el proceso educativo y para la posterior práctica profesional, por tanto el $70 \%$ de estos consideran que dicha utilización de las TIC les proporciona una adquisición de competencias tecnológicas. Mientras que el $80,8 \%$ las considera útiles para la posterior práctica profesional.

A continuación se procede a analizar y detallas el análisis de las variables de grado /especialidad y la valoración de los recursos TIC que utiliza el profesorado con nivel de confianza del 95\% $(\alpha=$ $0,95, \mathrm{p}<0,05)$, de manera que se muestran los siguientes resultados:
El 39,7\% del alumnado del grado de primaria manifiesta que las TIC no presentan dificultades en su manejo, verificándose, igualmente, que el $44,8 \%$ del alumnado de Primaria, se encuentra en algún grado de acuerdo en su mayoría, con que el profesorado utiliza las TIC para la distribución del contenido. Se destaca que el alumnado de Pedagogía no muestra en ningún porcentaje una valoración total de desacuerdo ante dicha situación, por lo que se deduce que en cierta medida, los docentes utilizan las herramientas tecnológicas para hacer llegar al alumnado el contenido que se debe utilizar en el proceso educativo. El $32,2 \%$ de los alumnos del grado de Primaria están en algún grado de acuerdo o totalmente de acuerdo con que el profesorado fomenta el uso de las TIC para llevar a cabo el proceso de comunicación, en contraste con el alumnado de Pedagogía que ninguno ha mostrado su total desacuerdo con dicha consideración. Destacamos que algo más de la mitad del alumnado encuestado, el 53,4\%, manifiesta su descontento, frente a un $46,6 \%$ que considera que el profesorado sí que enseña recursos tecnológicos para que el alumnado pueda utilizarlos en su futura práctica profesional. Cabe destacar al alumnado de grado de 
Primaria, en el que existe una proporción mayor de alumnos conformes con la afirmación anteriormente expuesta, frente a los demás grados que la valoran negativamente.

Según la valoración que hace el alumnado en función de si la utilización de las TIC en el proceso educativo de las diferentes asignaturas permite la adquisición de las competencias tecnológicas, se destaca que el alumnado perteneciente a tercer curso con un $24,7 \%$, se encuentra en algún grado de acuerdo, seguidos por los alumnos de segundo curso con un $24,1 \%$, que igualmente están de acuerdo. La inconformidad del alumnado de segundo curso, $7,7 \%$, se destaca ante la opinión de desacuerdo con que el uso de las TIC fomente la adquisición de competencias TIC.

Dimensión: Valoración del alumnado sobre organización, metodología y contenidos de las asignaturas

Finalizando con el último bloque en el que se valora la organización, la metodología utilizada y el contenido de las asignaturas mediante la utilización de las TIC por parte del profesorado, cabe destacar que el $84,4 \%$ de los alumnos evalúan positivamente que mediante la utilización de las TIC el contenido dispone de una mayor organización. El 92,9\% valora igualmente de manera positiva que posean un mejor y mayor acceso a la información. Esta mejora de la organización de los contenidos es posible, además, debido a los numerosos recursos de organización que las TIC ofrecen, como son calendarios o agendas electrónicas, las cuales un $73,7 \%$ de los alumnos valoran positivamente como útiles para la planificación de manera temporal de la tarea a realizar. El alumno contribuye de forma activa a la elaboración del contenido, esta elaboración se realizará a través de internet mediante diversas herramientas virtuales. El alumnado valora en un $64,5 \%$ de forma positiva este hecho. Cabe destacar que, a través de las TIC, el alumnado puede realizar un seguimiento sobre su evaluación, siendo valorado este hecho de manera positiva por el $60,1 \%$ de los encuestados, y que le permite modificar o mejorar su proceso de autoevaluación. El seguimiento que las TIC permiten que el alumno haga de su propia evaluación, permite que sean conscientes de sus progresos de manera que tomen decisiones sobre su aprendizaje. El 73,8\% de los encuestados se muestran de acuerdo y totalmente de acuerdo con esta aseveración, frente a un $26,2 \%$, que se muestra en algún grado en desacuerdo.

\section{Facilidad del alumnado de un seguimiento sobre su evaluación}

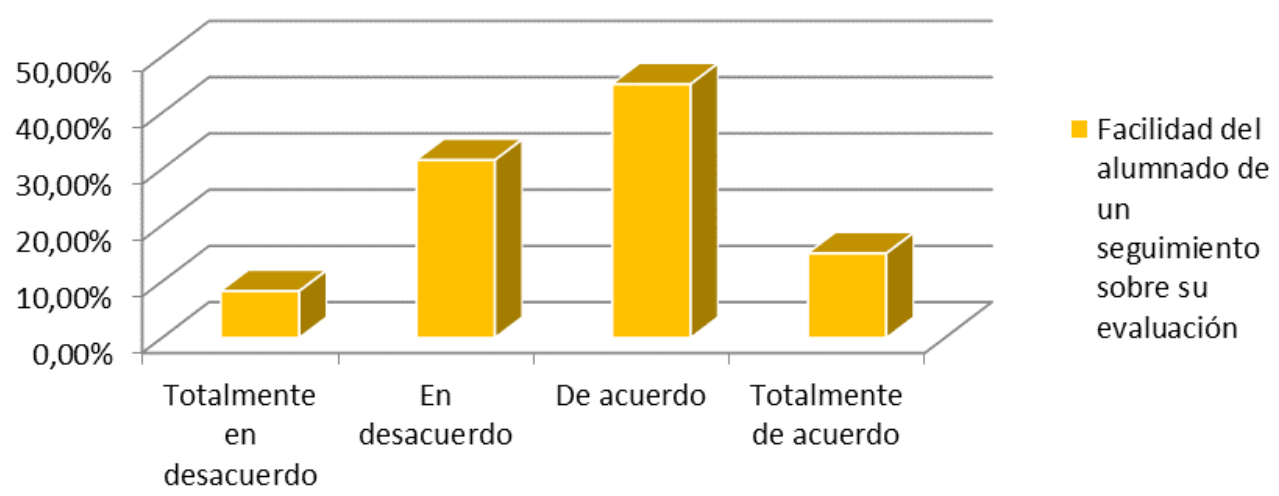

Gráfico 7: Valoración sobre la facilidad que ofrecen las TIC al alumnado para un seguimiento sobre su evaluación

En cuanto al análisis de contingencias de grado/especialidad y la valoración del alumnado sobre la organización, metodología y contenidos de las asignaturas, a nivel de confianza del $95 \%(\alpha=$ $0,95, \mathrm{p}<0,05)$, se muestra que el grado de primaria con un $43,7 \%$, presenta el mayor porcentaje que manifiesta su contento con que las actividades que se llevan a cabo a través de las TIC ponen en práctica lo aprendido. Por otro lado, el grado de
Pedagogía no manifiesta su descontento de ninguna forma con respecto a dicha situación. La mayor proporción de alumnado está de acuerdo o totalmente de acuerdo con la posibilidad de que las TIC ofrezcan potencial para poner en práctica lo aprendido en el proceso de enseñanza-aprendizaje. Con respecto al hecho de que a través de las TIC, el profesorado facilite al alumnado un seguimiento sobre su evaluación, el grado de Primaria es el que 
valora, en su mayoría, positivamente esta circunstancia con un $31,2 \%$, frente a un $24,4 \%$, que considera esta situación de forma negativa. De la misma forma, podemos, se constata, que el alumnado de segundo curso, el cual con un $26,3 \%$, se muestra de acuerdo o totalmente de acuerdo con que se realizan actividades prácticas a través de las TIC, frente al 5,3\% que muestran su descontento.

\section{DISCUSIÓN Y CONCLUSIONES}

El cambio de metodología es un aspecto importante valorado por el alumnado, considerado como necesario para la adaptación a la sociedad actual, estando igualmente de acuerdo ante la introducción de las TIC en el proceso educativo, sin olvidar el componente de presencialidad de profesorado y alumnado en dicho proceso. Las mejoras que se producen en el proceso de enseñanza-aprendizaje con la introducción de las TIC son variadas entre ellas la mejora de la calidad de aprendizaje; los resultados académicos; la comunicación (Sanabria y Hernández, 2011), debido a las numerosas herramientas virtuales que ofrecen las TIC para la mejora de la motivación del alumnado, proporcionando al alumnado un papel activo e innovador en el proceso educativo $y$ predisponiéndolo al aprendizaje (De Pablos Pons, 2007), siempre que el uso de las TIC no supongan una dificultad en su manejo, lo que desmotiva y dificulta el proceso de aprendizaje.

En cuanto a la falta de formación en herramientas virtuales por parte del profesorado y del alumnado, resulta una opinión extendida entre los participantes de la investigación (Barroso y Cabero, 2010), considerando necesaria una mejora de la formación en competencias tecnológicas. Con lo que respecta a la resistencia y uso deficiente por parte del profesorado en utilización e integración de TIC en el proceso educativo, se considera que existe un uso deficiente de las TIC por parte del profesorado, siendo herramientas básicas aquellas que utilizan en el proceso educativo. En cuanto a la resistencia del profesorado ante la integración de las TIC, los docentes manifiestan que es necesaria una adaptación al cambio de sociedad y exigencias marcadas.

Que el aprendizaje sea más práctico y significativo mediante el uso de las TIC, es un aspecto que se valora positivamente por el alumnado, permitiendo al alumno poner en práctica aquello que ha aprendido, implicándose en el proceso de manera activa, participando y teniendo cierto protagonismo en la construcción y aprendizaje del contenido (Ballesta et al, 2001). Es a través de las TIC que al alumnado le facilita la comprensión del contenido, posibilitando, igualmente, el aprendizaje de los contenidos, de manera que el alumno comprende las características básicas de la tarea y dispone de instrumentos que le sirven de apoyo en el proceso de aprendizaje rompiendo los clásicos escenarios formativos limitados a las instituciones permitiendo un aprendizaje activo y participativo contando con la orientación del docente. (Cabero, 2010). La comunicación, según el alumnado encuestado se ve reforzada con la utilización de las nuevas tecnologías, permitiendo un continuo flujo de información entre los mismos compañeros y con el profesor para el proceso de resolución de problemas, de apoyo o de distribución de la información, siendo este aspecto valorado positivamente entre el alumnado (Badía, 2006).

Las TIC proporcionan herramientas virtuales que permiten desarrollar competencias y habilidades relevantes para la futura práctica profesional y laboral, de manera que se traslada la necesidad de inculcarles conocimientos teóricos a inculcarles, además, habilidades como saber hacer, aprender a aprender y aprender a ser (Capella y Orts, 2010), permitiendo al alumno comprender aquello teórico que está aprendiendo a través de la relación con la realidad para una posterior puesta en práctica.

Es a pesar de la posibilidad de realizar un trabajo autónomo (Zabalza, 2004), el alumnado encuestado valora de igualmente forma positiva la posibilidad que ofrecen las TIC de realizar un trabajo colaborativo, intercambiando experiencias $\mathrm{y}$ opiniones (De Miguel, 2006).

Un porcentaje elevado del alumnado considera que el profesorado utiliza herramientas tecnológicas para la distribución del contenido, de manera que igualmente se utilizan para la exposición de los contenidos. El profesorado motiva la búsqueda de información y la investigación a través de la red, de igual manera, que el alumnado se siente motivado por parte del profesorado para la distribución de sus ideas y reflexiones fomentando, por tanto, el trabajo colaborativo (Marquès, 2001).

El alumnado valora de forma positiva que mediante el uso de las TIC se facilita una organización del contenido y comprensión de la tarea a realizar debido a dicha organización (Barberà, 2008). Las TIC posibilitan la realización de tareas prácticas (Santos et al, 2009), lo que permite una mejor comprensión de aquello a aprender. En lo referente al seguimiento por parte del alumnado de aquello que va aprendiendo, se 
valora que las TIC facilitan dicho proceso, permitiendo a su vez una retroalimentación, siendo consciente de su progreso (Cruz, 2005; Trujillo y Raso, 2010).

\section{REFERENCIAS}

Araujo, D., y Bermúdez, J. (2009). Limitaciones de las tecnologías de información y comunicación en la educación universitaria. Horizontes educacionales, (14)1, 9-24.

Badía, A. (2006). Ayuda al aprendizaje con tecnología en la educación superior. RUSC. Universities and Knowledge Society Journal, 3(2), 5-19.

Ballesta, F. J., Izquierdo, T., y Romero, B. E. (2011). Percepción del alumnado de pedagogía ante el uso de metodologías activas. Education siglo XXI, 29(2), 353368.

Ballesteros, B., Franco, D., y Carañana, J. P. (2012). El papel de las TIC en el EEES como motor para la transformación social. Disponible en: http://www.seeci.net/cuiciid/PDFs/INVESTIGACION\% 202.pdf

Barberà, E. (2008). Calidad de la enseñanza 2.0. RED: Revista de educación a distancia, 7(2), 1-17.

Barroso, J., y Cabero, J. (2010). Valoraciones de los alumnos sobre el E-learning en las universidades Andaluzas. EDUTEC revista electrónica de tecnología educativa, 31 .

Buendía, L. (2001). La Investigación por Encuesta. En L. Buendía et al. (Ed.). Métodos de Investigación en Psicopedagogía. Madrid: McGraw - Hill.

Cabero, J., Llorente, M. C., Leal, F., y Andrés, F. (2009). La alfabetización digital de los alumnos universitarios Mexicanos: una investigación de la universidad autónoma de Tamaulipas. Enseñanza \& Teaching, 27(1), 41-59.

Cabero, J. (2010). Retos de la integración de las TICs en los procesos educativos. Límites y posibilidades. Perspectiva educacional, 49(1), 32-61.

Capella, J.V., y Ors, R. (2010). Nuevo planteamiento metodológico orientado al aprendizaje y apoyado en el uso de las nuevas tecnologías para la docencia en la universidad del siglo XXI. Recuperado a partir de http://reposital.cuaed.unam.mx:8080/jspui/bitstream/12 3456789/2547/1/118.pdf

Cruz, A. (2005). Seguimiento académico del alumnado. En Benito, A. Nuevas claves para la docencia universitaria en el espacio europeo de educación superior. Madrid: Narcea.

De Juanas, A., y Fernández, P. (2008). Competencias y estrategias de aprendizaje. Reflexiones sobre el proceso de cambio en el EEES. Cuadernos de trabajo social, 21, 217-230

De la Cruz, A. (2003). El proceso de convergencia europea: ocasión de modernizar la universidad española si se produce un cambio de mentalidad en gestores, profesores y estudiantes. Aula abierta, 82, 191-216.

De Miguel, M. (2006). Modalidades de enseñanza centradas en el desarrollo de competencias: orientaciones para promover el cambio metodológico en el Espacio Europeo de Educación Superior. Recuperado a partir de http://www.enlinea.ugfca.net/enlinea/pluginfile.php/202 85/mod_resource/content/1/LIBRO\%20MARIO\%20DE $\%$ 20MIGUEL.pdf
De Pablos, J. (2007). El cambio metodológico en el Espacio Europeo de Educación Superior y el papel de las tecnologías de la información y la comunicación. RIED, 10(2), 15-44.

Ferro, C. A., Martínez, A. I., \& Otero, M. del C. (2009). Ventajas del uso de las TICs en el proceso de enseñanza-aprendizaje desde la óptica de los docentes universitarios españoles. Edutec: Revista electrónica de tecnología educativa, 29, 1-12.

Fox, D. J. (1981). El proceso de investigación en educación. Ediciones universidad de Navarra.

Marquès, P. (2001). Algunas notas sobre el impacto de las TIC en la universidad. En Educar, 28, 83-98.

Rodríguez, R. (2011). Repensar la relación entre las TIC y la enseñanza universitaria: problemas y soluciones. Profesorado: Revista de currículum y formación del profesorado, 15(1), 9-22.

Salinas, J. (1998). Redes y desarrollo profesional del docente: entre el dato serendipiti y el foro de trabajo colaborativo. Profesorado: Revista de curriculum y formación del profesorado, 2(1), 13-24.

Sanabria, A., y Hernández, C. M (2011). Percepción de los estudiantes y profesores sobre el uso de las TIC en los procesos de cambio e innovación en la enseñanza superior. Revista de psicología, 29, 273-290.

Santos, J., Galán, J., Izquierdo, L., y Olmo, R. (2009). Aplicaciones de las TIC en el nuevo modelo de enseñanza del EEES. Dirección y Organización, 39, 511

Shaughnessy, J. J., Zechmeister, E. B., y Zechmeister, J. S. (2007). Métodos de investigación en psicología. México: McGraw-Hill.

Trujillo, J. (2006). Un nuevo currículum: tecnologías de la información en el aula. Educación y educadores, 9(1), 161-174.

Trujillo, J. M., y Raso, F. (2010). Formación inicial docente y competencia digital en la convergencia europea (EEES). Enseñanza \& Teaching, 28(1), 49-77.

Valcárcel, M. (Coord.) (2003). La preparación del profesorado universitario español para la convergencia europea en educación superior. Recuperado a partir de http://campus.usal.es/ ofeees/ESTUDIOS_INFORMES _GRALES/informe_final.pdf

Zabalza, M. (2004). Guía para la planificación didáctica de la docencia universitaria en el marco del EEES. Recuperado a partir de http://www.upcomillas.es/eees/Documentos/guiaplan.pd 